\title{
State and Trait Anxiety of Adolescents with Autism Spectrum Disorders
}

\author{
So Yoon Kim¹, Young Ah Kim², Da-Yea Song², Guiyoung Bong², \\ Jong-myeong $\mathrm{Kim}^{2}$, Joo Hyun $\mathrm{Kim}^{2}$, and Hee Jeong $\mathrm{Yoo}^{2,3} \bowtie$ \\ 1'Department of Teacher Education, Duksung Women's University, Seoul, Republic of Korea \\ ${ }^{2}$ Department of Psychiatry, Seoul National University Bundang Hospital, Seongnam, Republic of Korea \\ ${ }^{3}$ Department of Psychiatry, Seoul National University College of Medicine, Seoul, Republic of Korea
}

\begin{abstract}
Objective This study examined how state and trait anxiety of adolescents with autism spectrum disorders (ASD) are associated with their demographic characteristics, repetitive and restricted behaviors (RRBs), and internalizing and externalizing problem behaviors.

Methods A total of 96 participants with ASD (mean age=14.30 years; 91 males) completed a battery of tests including the State/Trait Anxiety Inventory (STAI), the Autism Diagnostic Interview-Revised, the Social Responsiveness Scale (SRS), and a cognitive test measuring intelligence quotient (IQ). Participants' parents completed the Child Behavior Checklist (CBCL). Pearson's correlations among age, IQ, two subscales of the STAI (i.e., STAIS and STAIT, measuring self-reported state and trait anxiety, respectively), and the Anxiety subscale of CBCL (i.e., CBCL-Anxiety, measuring parent-reported trait anxiety) were computed. Subsequently, Pearson's correlations were computed among the three anxiety measures, RRBs, and problem behaviors, while controlling for participants' age and IQ.

Results The STAIS and CBCL-Anxiety were both significantly correlated with higher age, sensory sensitivity, depressive symptoms, somatic complaints, and aggressive behaviors. All three anxiety variables were significantly and positively correlated with total SRS RRB scores. Additionally, the STAIS and STAIT were significantly associated with more severe Compulsion/Adherence behaviors, and the CBCL-Anxiety was also significantly associated with more severe Rule-breaking Behaviors.

Conclusion Self-reported state anxiety showed association patterns similar to those of parent-reported trait anxiety. Future studies investigating the precise operationalization of different anxiety instruments are needed to accurately measure the anxiety of adolescents with ASD.

Psychiatry Investig 2021;18(3):257-265
\end{abstract}

Key Words Anxiety, Problem behavior, Autism spectrum disorders, Repetitive behaviors, Adolescence.

\section{INTRODUCTION}

Anxiety is considered to be one of the most common comorbidities of autism spectrum disorder (ASD). ${ }^{1-4}$ Approximately $40 \%$ of youths with ASD meet the diagnostic criteria of an anxiety disorder, ${ }^{5}$ and children with ASD are at significantly increased risk of having anxiety disorders than children without ASD. ${ }^{6}$ Such tendencies have been reported to negatively influence the social participation, ${ }^{7}$ life satisfaction, ${ }^{8}$

Received: August 28, 2020 Revised: November 16, 2020

Accepted: December 18, 2020

$\triangle$ Correspondence: Hee Jeong Yoo, MD, PhD

Department of Psychiatry, Seoul National University Bundang Hospital, Seoul National University College of Medicine, 82 Gumi-ro 173beon-gil, Bundang-gu, Seongnam 13620, Republic of Korea

Tel: +82-31-787-7436, Fax: +82-31-787-4058, E-mail: hjyoo@snu.ac.kr

(c) This is an Open Access article distributed under the terms of the Creative Commons Attribution Non-Commercial License (https://creativecommons.org/licenses/bync/4.0) which permits unrestricted non-commercial use, distribution, and reproduction in any medium, provided the original work is properly cited. and long-term psychological health of individuals with ASD. ${ }^{9}$ Researchers have attempted to identify the factors that may be associated with anxiety such as demographic characteristics, ASD symptoms, and problem behaviors to develop an effective intervention targeting this particularly vulnerable population.

Various demographic factors such as intelligence quotient (IQ), age, and gender have been shown to influence the anxiety levels of individuals with ASD. A recent meta-analysis reported that youths with ASD have higher anxiety levels than clinically referred children, and this difference increased with age. Additionally, differences in anxiety levels between children with ASD and typically developing (TD) children have been reported to increase with IQ. ${ }^{10}$ Vasa and Mazurek ${ }^{11}$ speculate that individuals with higher IQs may be more aware of their social and adaptive difficulties, resulting in higher anxiety levels. On the other hand, studies of the effects of gender 
have not yielded consistent results. Gotham et al..$^{12}$ reported that females with ASD have higher anxiety levels than males with ASD, while others reported no gender differences. ${ }^{13,14}$

Among the ASD symptoms, restricted and repetitive behaviors (RRBs) have been shown to be consistently associated with anxiety levels in individuals with ASD. ${ }^{9,13,15,16}$ As one of the core diagnostic characteristics of ASD, RRBs include 1) stereotyped and repetitive motor movements and manipulation of objects; 2) restricted and fixated interests; 3) hyperor hypo-reactivity to sensory input; and 4) compulsive adherence to routines and ritualized patterns of verbal and nonverbal behaviors that occur regularly and interfere with daily functioning. ${ }^{17} \mathrm{RRB}$ severity at the time of ASD diagnosis (ages 2-5) was reported to be a risk factor for future anxiety symptoms at ages 8-11 years. ${ }^{15}$ Among RRBs, higher levels of insistence on sameness, ${ }^{9}$ increased sensory sensitivity, ${ }^{18,19}$ and restricted interests (intense preoccupation with limited patterns of activities or arrangements of things $)^{20}$ were significantly associated with anxiety in children with ASD. However, the causal pathways underlying the associations between RRBs and anxiety in ASD have not been identified. For instance, some researchers contend that RRBs (e.g., repetitive thoughts) result in anxiety, ${ }^{9}$ while others argue that anxiety causes an increase of RRBs. ${ }^{20,21}$

Anxiety has also been associated with internalizing (e.g., depression) and externalizing (e.g., oppositional attitudes or physical aggression) behavioral problems in children and adolescents with ASD. ${ }^{22-24}$ Some researchers contend that teaching adaptive strategies to relieve anxiety may decrease problem behaviors. ${ }^{23}$ However, arguments for the opposite direction of cause and effect have also been raised. For instance, Niditch et al. ${ }^{25}$ argue that aggressive behaviors may lead to increased anxiety due to peer rejection ${ }^{26}$ or by instigating the constant state of anxiety from behaving anti-socially. ${ }^{27}$

The above-mentioned studies that investigate the factors associated with the anxiety of adolescents with ASD tend to utilize instruments that measure trait anxiety (i.e., relatively stable, general predisposition of anxiety proneness). Yet, researchers studying anxiety contend that there is another distinct dimension of anxiety, which is state anxiety (i.e., a transitory or situational feeling of tension and apprehension accompanied by physiological arousal)..$^{28}$ Trait and state anxiety seem to be operating in different patterns in adolescents with ASD. For instance, Mertens et al. ${ }^{29}$ demonstrated that while the trait anxiety of adolescents with ASD was higher than that of TD adolescents, there was no group difference in state anxiety, measured by skin conductance responses and heart rate variability. However, with the majority of the current literature focusing on trait anxiety, very few studies have specifically examined the self-reported state anxiety of indi- viduals with ASD; the associations between state anxiety and different individual factors of adolescents with ASD remain largely unknown.

This study, therefore, aimed to better operationalize the anxiety in adolescents with ASD by investigating how trait and state anxiety are distinctively associated with demographic factors, RRBs, and problem behaviors. In line with the results from van Steensel and Heeman's ${ }^{10}$ meta-analysis and previous literature, we hypothesized that trait anxiety would be positively correlated with higher age, higher IQ, more severe RRBs, and more internalizing and externalizing problem behaviors. We did not have a hypothesis regarding the state anxiety in ASD due to the lack of literature.

\section{METHODS}

\section{Participants}

This study is a secondary analysis of previously collected data from a social skills training group intervention for adolescents and youths with ASD. ${ }^{30}$ In the original study, participants were screened out if they had 1) a verbal IQ score lower than 65 on the Wechsler Adult Intelligence Scale (WAISIV), Korean Educational Development Institute-Wechsler Intelligence Scale for Children-Revised (KEDI-WISC), or the Wechsler Intelligence Scale for Children (WISC-IV); 2) a history of major mental illness (e.g., schizophrenia or bipolar disorder), 3) severe oppositional and aggressive tendencies; or 4) hearing, visual, or physical disabilities or neurological illnesses that would prevent participation in the intervention program. Among 110 participants who attended the social skills intervention, 14 were excluded from this study because we did not have data to confirm their diagnosis.

A total of 96 participants with ASD [age range $=11-18$ yearsold; mean age (standard deviation) $=14.30$ years $(1.80) ; 91$ males] were included in this study. Detailed demographic characteristics are reported in Table 1. All data collection procedures (IRB no: B-1108/133/007, B-1301/187-006, B-1106/ 129-009) and retrospective analyses of collected data (IRB no: B-2001/591-103) were approved by the Institute Review Board (IRB).

\section{Study procedures}

Participants were recruited from the child and adolescent psychiatric clinics of the corresponding author's institution and from advertisements mailed to child psychiatrists registered in the Korean Academy of Child and Adolescent Psychiatry or shared on online parent support group blogs. Once the participants and their parents had given their informed consent, the Korean translation of the Autism Diagnostic Observation Schedule-Second Edition (ADOS-2) ${ }^{31,32}$ the Ko- 
Table 1. Participant characteristics

\begin{tabular}{lcl}
\hline Demographic characteristics & Mean (SD) & Range \\
\hline Age (years) & $14.30(1.80)$ & {$[11,19]$} \\
FSIQ & $98.91(16.05)$ & {$[68,136]$} \\
ADI-R current score-SI & $9.64(4.27)$ & {$[0,19]$} \\
ADI-R current score-C & $7.33(3.30)$ & {$[0,16]$} \\
ADI-R current score-RRB & $3.27(2.05)$ & {$[0,9]$} \\
SCQ & $23.79(6.49)$ & {$[2,32]$} \\
SRS-2 & $87.70(27.80)$ & {$[27,172]$} \\
CBCL-Anxiety & $66.84(9.51)$ & {$[50,100]$} \\
STAIT & $38.05(9.11)$ & {$[20,57]$} \\
STAIS & $38.54(9.01)$ & {$[20,57]$} \\
\cline { 2 - 3 } & \multicolumn{2}{c}{ Percentage of male } \\
\cline { 2 - 3 } Gender
\end{tabular}

FSIQ: Full scale intelligence quotients, SD: standard deviation, ADI$\mathrm{R}$ : autism diagnostic interview-revised, SI: social interaction, C: communication, RRB: Restricted Repetitive Behavior, SCQ: Social Communication Questionnaire, SRS-2: Social Response Scale-2, CBCL: Child Behavior Checklist, STAIT: State-Trait Anxiety Inventory-Trait, STAIS: State-Trait Anxiety Inventory-State

rean translation of the Autism Diagnostic Interview-Revised (ADI-R), ${ }^{33,34}$ and the WAIS or WISC were administered during their one-time visit to the authors' laboratory to screen for eligibility before the intervention. Research-reliable researchers (i.e., trained professionals who are qualified to implement the ADOS-2 and/or ADI-R) administered the ADOS- 2 and ADI-R, and trained graduate students administered and scored the WAIS or WISC under the close supervision of clinical psychologists. All participants scored higher than the cutoff points in both the ADOS-2 and ADI-R diagnostic algorithms. In addition, all caregivers of participants completed a battery of surveys including the Social Communication Questionnaire (SCQ) ${ }^{35}$ and the Social Responsiveness Scale-Second Edition (SRS-2) ${ }^{36}$ to confirm the participants' diagnoses. The ASD diagnoses of all participants were confirmed by psychiatrists' best clinical judgments based on their ADOS-2, ADI-R, SCQ, and SRS-2 scores.

A few weeks later, eligible participants came back to participate in the intervention. After the first session of the intervention, participants and caregivers completed the State/Trait Anxiety Inventory (STAI ${ }^{37}$ and the Child Behavior Checklist for Ages 6-18 (CBCL/6-18). ${ }^{38}$ See Yoo et al. ${ }^{30}$ for a detailed account of the recruitment and screening procedures and detailed information about the intervention.

\section{Measures}

\section{STAI $^{37}$}

The $\mathrm{STAI}^{37}$ is a self-report questionnaire measuring trait and state anxiety. It consists of two 20-item subscales: the STAIT, which measures how the respondents generally feel (i.e., trait anxiety), and the STAIS, which measures how the respondents feel at a given moment (i.e., state anxiety). Items are measured on a 4-point Likert scale with a higher score representing higher anxiety. Both subscales have been shown to have good internal reliability, test-retest reliability, and convergent and discriminant validity for youths. ${ }^{37}$ The reliability and validity of the Korean translation of the STAI have been reported to be sufficient (test-retest reliability: $r=0.69$ for STAIS and $\mathrm{r}=0.75$ for the STAIT; internal consistency: Cronbach's alpha 0.88 for the STAIS and Cronbach's alpha $=0.83$ for the STAIT) ${ }^{39}$ Cronbach's alphas from the current study were 0.91 for the STAIS and 0.90 for the STAIT.

\section{$\mathrm{CBCL} / 6-18^{38}$}

The K-CBCL/6-18 is a 120 -item parent rating scale designed to assess behavioral issues during the past six months in children ages 6 through 18. The CBCL has been reported to have sufficient validity and reliability. ${ }^{38,40}$ The Korean standardized version of the CBCL 6-18 ${ }^{41}$ was used in this study. The CBCL consists of two dimensions: DSM-Oriented Scales and Syndrome Scales. The DSM-Oriented Scales of the CBCL were constructed based on agreement among experts' ratings of the items' consistency with Diagnostic and Statistical Manual-IV (Text Revision) diagnostic criteria. The Syndrome Scales were derived via factor analysis of existing items and consisted of Anxious/Depressed, Withdrawn/Depressed, Somatic Complaints, Social Problems, Thought Problems, Attention Problems, Rule-breaking Behavior, and Aggressive Behavior scales. Combining the scores of Anxious/Depressed, Withdrawn/Depressed, and Somatic Complaints yields Internalizing summary scores, and combining Rule-Breaking and Aggressive Behavior yields Externalizing Problem summary scores. This study individually utilized the Withdrawn/Depressed and Somatic Complaints scales to represent internalizing behaviors and the Rule-breaking Behavior and Aggressive Behavior scales to represent externalizing behaviors.

The Anxiety score of the DSM-Oriented Scales (CBCLAnxiety) was used in this study as another trait anxiety variable. We did not use the Anxious/Depressed score in the Syndrome Scales because it simultaneously captured the depressive symptoms, which was used as one of the variables of interest. We did not include other DSM-Oriented Scales in the analysis to limit the number of hypotheses tested and avoid the Type 1 errors from multiple hypothesis testing. Cronbach's alphas of subscales used in this study ranged from 0.70 to 0.88 .

\section{Autism Diagnostic Interview-Revised (ADI- ${ }^{33}$ )}

The ADI-R is a semi-structured diagnostic interview ad- 
ministered to caregivers. With 93 items, trained examiners rate each question with a higher score suggesting more severe ASD-related symptoms. A diagnostic algorithm composed of selected items generates three domains: social interaction, communication, and RRBs. The RRB domain can be further split into subdomains: 1) encompassing preoccupation or circumscribed pattern of interests, 2) compulsive adherence to routines or rituals (labeled as compulsive adherence hereafter), 3) motor mannerisms, and 4) preoccupation with parts of objects or non-functional elements of the material. Participants' current scores of total RRBs, the four domains of RRBs, and the sensory sensitivity score (i.e., combined score of the three ADI-R items that ask participants' likes and dislikes of particular sensory stimuli) were utilized in this study as variables of interests. With approval by Western Psychological Services, the Korean translated version of the ADI- $\mathrm{R}^{34}$ was used in this study.

\section{Social Responsiveness Scale-2 (SRS-2 ${ }^{36}$ )}

The SRS-2 is a parent-report questionnaire used to measure ASD symptomatology. Based on a 4-point scale, the SRS-2 is composed of 65 items grouped into five scales: Social Awareness, Social Cognition, Social Communication, Social Motivation, and Autistic Mannerisms. Ratings are converted to tscores with higher scores indicating more severity in ASD symptoms. Having previously been administered in ASD studies as a diagnostic measure, ${ }^{42}$ the SRS- 2 has good internal consistency and concurrent and discriminant validity. ${ }^{36}$ The Autistic Mannerism subscale was used as a measure of RRB in this study, and Cronbach's alpha of the Mannerism subscale from the current study was 0.92 . The approved translated version of the SRS- 2 by the WPS Publishers was used in this study.

\section{Autism Diagnostic Observation Schedule-second edition (ADOS-2 $2^{31}$ )}

The ADOS-2 is a semi-structured ASD diagnostic instrument that is based on direct observations by a trained expert. The ADOS-2 includes five modules that are composed of different tasks to accommodate developmental age and expressive language skills. Scoring is based on the presence or absence of behaviors and social interactions shown throughout the assessment with higher ratings suggesting greater impairment. The sum of the converted scores must meet both cutoffs in the Social Affect and RRB domains for an individual to be classified as having autism. All participants were assessed with either Module 3 or 4 because they were verbally fluent adolescents or youths. We did not include the RRB scores of the ADOS- 2 as a variable in this study because the short interaction with the examiner might not be enough to fully cap- ture the extent and range of participants' RRBs. ${ }^{43}$ Cronbach's alphas of Module 3 and 4 from the current study were 0.67 and 0.79 , respectively.

\section{KEDI-WISC ${ }^{44}$, WISC-IV ${ }^{45}$ and WAIS-IV ${ }^{46,47}$}

The KEDI-WISC and the Korean versions of the WAIS-IV and WISC-IV, which are cognitive assessments designed to measure intelligence and cognitive ability, were used to yield full-scale intelligence quotients (FSIQ) of participants. ${ }^{44,46,48}$ The KEDI-WISC was used before the Korean version of the WISC-IV was available, and the decision to use WISC-IV/KEDI-WISC or WAIS-IV was made based on participants' age.

\section{Data analysis}

First, the means, standard deviations of, and zero-order correlations among the STAIT, STAIS, and CBCL-Anxiety were computed. The equality of the correlation coefficients of the STAIT and CBCL-Anxiety and of the STAIS and CBCL-Anxiety was tested, using the "cortesti" function (i.e., a function that allows the approximation of whether a certain correlation strength is different from the strength of another correlation) in Stata statistical software: Release 15 (College Station, TX, USA). ${ }^{49}$ Then, pairwise Pearson $r$ correlations among STAIS, STAIT, CBCL-Anxiety, age, and FSIQ were calculated. Subsequently, partial correlations among the STAIT, STAIS, CBCL-Anxiety, total RRB algorithm score from the ADI-R, and total scores from the SRS Mannerism subscales were computed, while controlling for age and FSIQ. To identify the specific types of RRBs associated with anxiety, we conducted partial correlations among STAIT, STAIS, CBCLAnxiety, the four subdomain scores of the RRB domain, and the sensory sensitivity variable of ADI-R. Sensory sensitivity was calculated by adding the three ADI-R items that capture participants' atypical responses to sensory stimuli. Finally, the partial correlations among Withdrawn/Depressed, Somatic Complaints, Rule-Breaking, Aggressive Behavior subscales of the CBCL, STAIT, STAIS, and CBCL-Anxiety were computed, while controlling for age and FSIQ.

\section{RESULTS}

The means and standard deviations of the STAIS, STAIT, and CBCL-Anxiety are included in Table 1. The magnitude of the correlation coefficient between the STAIT and CBCLAnxiety was not significantly different from that between the STAIS and CBCL-Anxiety, $\mathrm{p}=0.62$.

Benchmarks outlined by Cohen ${ }^{50}$ were used to categorize the magnitudes of the significant effect sizes as 'small' (0.10$0.29)$, 'medium' (0.30-0.49) and 'large' $(\geq 0.50)$. The age variable was significantly correlated with the STAIS and CBCL- 
Anxiety, $r=0.31, p=0.002$ (i.e., representing a medium effect size) and $r=0.24, p=0.02$ (i.e., representing a small effect size), respectively, but not with the STAIT, $r=0.19, p=0.07$ ( $n=96$ for all three effect sizes). The FSIQ was not significantly correlated with the STAIS, STAIT and CBCL-Anxiety, $\mathrm{r}=0.05, \mathrm{p}=0.61$, $r=-0.05, p=0.66$, and $r=-0.14, p=0.19$ respectively $(n=90$ for all three effect sizes).

Table 2 presents the partial correlation matrix among the STAIS, STAIT, CBCL-Anxiety, RRBs, problem behavior variables, after taking age and IQ into account. Results of zeroorder correlational analyses without controlling for age and FSIQ are presented in Supplementary Table 1 (in the onlineonly Data Supplement).

Among the RRB variables, the total SRS Mannerism score was significantly associated with all three anxiety variables. The effect sizes for the STAIS and STAIT were small $(r=0.29)$, while the effect size for CBCL-Anxiety was large ( $r=0.50)$. The total RRB algorithm score from the ADI-R was not associated with any of the three. However, among the domain scores of the ADI-R, compulsive adherence was significantly associated with the STAIS and STAIT with small effect sizes (both rs=0.24), but not with the CBCL-Anxiety. Additionally, sensory sensitivity variables were significantly associated with the STAIS and CBCL-Anxiety with small $(r=0.24)$ and medium $(r=0.34)$ effect sizes, respectively.

The STAIS was significantly correlated with Withdrawn/ Depressed, Somatic Complaints, Aggressive Behavior with effect sizes ranging from small to medium $(r=0.24-0.37)$. The

Table 2. Correlations matrix controlling for age and IQ

\begin{tabular}{lccc}
\hline \multicolumn{1}{c}{ Variables } & STAIS & STAIT & CBCL-anxiety \\
\hline Withdrawn/depressed & $0.37^{\dagger}$ & 0.20 & $0.61^{\dagger}$ \\
Somatic complaints & $0.26^{*}$ & 0.19 & $0.42^{\dagger}$ \\
Rule-breaking behavior & 0.10 & -0.001 & $0.26^{*}$ \\
Aggressive behavior & $0.24^{*}$ & 0.14 & $0.24^{*}$ \\
SRS-2 mannerism & $0.29^{\dagger}$ & $0.29^{\dagger}$ & $0.50^{\dagger}$ \\
RRBs total scores & 0.12 & 0.11 & 0.06 \\
from the ADI-R & & & \\
ADI-R 1 & -0.06 & -0.11 & -0.06 \\
ADI-R 2 & $0.24^{*}$ & $0.24^{*}$ & 0.12 \\
ADI-R 3 & 0.01 & 0.12 & -0.02 \\
ADI-R 4 & 0.10 & 0.03 & 0.19 \\
Sensory sensitivity & $0.24^{*}$ & 0.20 & $0.34^{\dagger}$ \\
\hline
\end{tabular}

$\mathrm{N}=90$ for all correlations. ${ }^{*} \mathrm{p}<0.05,{ }^{\dagger} \mathrm{p}<0.01$. ADI-R 1 : encompassing preoccupation or circumscribed pattern of interest, ADI-R 2: compulsive adherence to routines or rituals, ADI-R 3: motor mannerisms, ADI-R 4: Preoccupation with parts of objects or non-functional elements of material, STAIT: State-Trait Anxiety InventoryTrait, STAIS: State-Trait Anxiety Inventory-State, CBCL: Child Behavior Checklist, ADI-R: autism diagnostic interview-revised, IQ: intelligence quotient
STAIT was not significantly correlated with any of the externalizing and internalizing behaviors. The CBCL-Anxiety was significantly associated with all four problem behavior scales of the CBCL with the effect sizes ranging from small to large $(\mathrm{r}=0.24-0.61)$.

\section{DISCUSSION}

The findings showed that state anxiety and trait (self- and parent-reported) anxiety of youths with ASD were associated with different demographic and behavioral variables. State anxiety was associated with age, depressive symptoms, somatic symptoms, aggressive behaviors, the total RRB scores from the SRS, the Compulsion/Adherence, and Sensory Sensitivity. As partial confirmation of the initial hypothesis, selfreported trait anxiety was associated with the total SRS RRB scores and the Compulsion/Adherence. Parent-report trait anxiety was associated with age, all problem behaviors, the total SRS RRB scores, and Sensory Sensitivity. Following is a discussion of the potential rationale for the identified significant associations.

\section{Age and IQ}

First, age was associated with state anxiety but not with selfreported trait anxiety, showing that trait anxiety is a relatively stable proneness ${ }^{51}$ while state anxiety measures are subject to change as children grow up. van Steensel and Heeman ${ }^{10}$ contend that as children with ASD get older, they encounter higher social expectations to adapt to their surroundings and consequently become more aware of their own social difficulties, leading to more stress and anxiety. Considering that state anxiety was measured after the first group intervention session during which participants were introduced to and asked to interact with unfamiliar peers, the state anxiety score could have reflected the participants' individual variations in cognitive and affective responses to the socially demanding situations. It has been previously suggested that unfamiliarity of the intervention research situations may increase the self-report state anxiety of participants. ${ }^{52}$ In this case, the state anxiety of older participants, who were likely to be more aware of the social demands during the intervention session than younger participants, may have been particularly heightened. As the validity and reliability of the STAI have been established under relatively neutral, non-anxiety provoking contexts in previous studies, future studies should explore how the reliability of the STAIS may vary depending on the context of the situation in which the measure is taken.

With respect to the parent-report anxiety, parents may expect their older children to manage their anxiety more effectively and, therefore, show less anxious behaviors. This in- 
creased expectation may lead the parents to over-rate the severity of their older children's anxiety even if the children are in fact showing the same level of anxiety regardless of their age. At the same time, it is also possible that older children do experience more anxiety, and parents are accurately reporting their children's anxiety levels.

Unlike the findings from van Steensel and Heeman ${ }^{10}$ and contrary to our hypothesis, IQ was not associated with any of the anxiety variables in this study. Although we included participants with a relatively wide IQ range of 68-136, we cannot rule out the possibility that different association patterns may emerge when more participants with lower IQ levels (e.g., <70) are included.

\section{RRBs}

All three anxiety variables were associated with the total RRB scores measured by the SRS, but not with the total algorithm score of the ADI-R, partially confirming our hypothesis. We speculate that the total RRB scores of the ADI-R, which are measured by six items, may not have been enough to capture items that are particularly more linked with anxiety. For instance, we found items related to sensory sensitivity to be significantly linked with state anxiety. However, according to the ADI-R diagnostic algorithm, a participant's score is based on the selection of a higher rating between "interests in part and/or non-functional parts" and "sensory oversensitivity." If one scored higher in the former question, their total RRB domain score would not have captured constructs related to sensory sensitivity. This calls for the need to use instruments that target specific and nuanced constructs within RRBs in future research. For instance, anxiety in ASD was reported to be associated with specific constructs within RRBs such as insistence on sameness 1 and intolerance to uncertainty (IU). ${ }^{53,54}$ Future researchers should conduct follow-up studies with instruments that can validly and directly measure these constructs and examine how different aspects of RRBs are associated with state and trait anxiety.

The significant associations between self-reported trait and state anxiety and the Compulsion/Adherence subscale of the ADI-R mirror the results of Hallet et al., ${ }^{55}$ who found that the obsessive-compulsive symptoms of ASD were associated with anxiety for participants with IQ $>70$. Gillott et al. ${ }^{56}$ regarded anxiety as both the source and the consequence of the ritualistic and compulsive behaviors of ASD. That is, individuals with ASD may engage in ritualistic or compulsive behaviors to relieve their anxiety ${ }^{57}$ and may also become anxious because social environments are not organized to accommodate their compulsive and ritualistic behaviors.

In terms of the significant associations between state anxiety and parent-reported trait anxiety reported in this study, individuals with high sensory sensitivity may be constantly bothered by various sensory stimuli in the environment and may attempt to regulate the negative, overwhelming emotions with maladaptive strategies such as avoidance or withdrawal. ${ }^{58,59}$ Trying to avoid various sensory stimuli may require individuals to be alert to their environment and ultimately lead to an increased likelihood of state anxiety. ${ }^{60-62}$ Green et al. ${ }^{63}$ also found that sensory oversensitivity predicts anxiety symptoms one year later. Meanwhile, Wigham et al. ${ }^{19}$ suggested that IU was a mediator between sensory under- and over-responsiveness and anxiety in children with ASD. Individuals with ASD with high IU may find facing and interpreting aversive and unpredictable sensory stimuli to be stressful and anxious. $^{53,54}$

\section{Problem behaviors}

State anxiety was associated with all problem behaviors except for rule-breaking behaviors. Youths with ASD who experience high state anxiety during stressful situations may be engaging in problem behaviors as maladaptive strategies to resolve the anxiety at the moment. ${ }^{22}$ It is also possible that youths with ASD who engage in frequent problem behaviors may end up in anxiety-provoking situations and, therefore, stay in higher levels of state anxiety more often than youths with fewer problem behaviors. Moreover, contrary to our initial hypotheses, self-reported trait anxiety was not associated with any of the internalizing and externalizing behavior symptoms in youths with ASD, suggesting that self-reported state anxiety during stressful situations may be more strongly implicated with problem behaviors compared to self-reported trait anxiety.

\section{Implications}

The findings of this study bear several implications for future studies. To our knowledge, this is the first study that examined the associations between the self-reported state anxiety of adolescents with ASD and their individual and behavioral variables. Future studies should complement the findings of this study by comparing the association patterns between when state anxiety is measured by a self-report instrument and when measured by behavioral or physiological measures (e.g., electroencephalogram in Van Hecke et al. ${ }^{64}$; electrodermal activity in Jain et al. ${ }^{65}$; and heart rate in Mertens et al. ${ }^{29}$ ).

Except for the Rule-Breaking and Compulsion/Adherence, parent-report trait anxiety was associated with the same variables with which self-report state anxiety variables were associated, while the parent-reported and self-reported trait anxiety measures demonstrated markedly different patterns of associations with the purported variables of interest. These patterns may have emerged because parents are usually mak- 
ing judgments about their children's trait anxiety levels based on how youths express their anxiety in taxing situations, and these may be better captured in self-report state anxiety items (e.g., "I feel jittery") than in trait anxiety items (e.g., "I am a steady person"). Additionally, given the high prevalence rate of alexithymia (i.e., difficulty in identifying and describing emotions) in ASD, ${ }^{66}$ we speculate that the self-reported trait anxiety, which requires objective and long-term self-reflection and awareness of emotions and moods, may not be as accurate as the parent-reported trait anxiety. Moreover, the association patterns of parent-reported trait anxiety may be similar to those of self-reported state anxiety because reporting how anxious they feel at the moment may be easier for adolescents with ASD than reporting on their general proneness.

Previous studies have not reached a consensus on whether parent-report can accurately measure the anxiety levels of adolescents and youths with ASD. Some studies contend that parents may not be able to accurately judge their children's internalizing symptoms because adolescents with ASD may be hiding their emotional distress from their parents. ${ }^{67}$ Others argued that their lack of a theory of mind abilities ${ }^{42}$ makes it difficult for individuals with ASD to accurately assess their anxious states and questions the reliability of their self-report results. We urge further research on the assessments of anxiety captured by self- and parent-reports, perhaps using qualitative methods (e.g., cognitive interview) to explore in detail the reasons for their responses to each item and how different contextual variables influence the constructs captured in the items. Moreover, we suggest that future studies measuring the adolescents' trait anxiety should utilize both self- and parent-reports to gather multi-faceted, objective, and accurate information.

Conner et al. ${ }^{52}$ showed that parents' own state and trait anxiety symptoms were significantly associated with parentreported anxiety and problem behaviors of their adolescent children with ASD. Noting that these anxiety levels were measured before the anxiety intervention of the adolescents with ASD, Connor et al. ${ }^{52}$ explained that parents with high anxiety levels may have been more anxious themselves due to the timing of the measurement, and this anxiety may have influenced parents' interpretation of their children's behaviors. The same mechanism may have also applied to this study. This underscores the need for studies examining the association between parents' own anxiety in various anxiety-provoking situations and how they report about their child's anxiety.

While Kim et al. ${ }^{23}$ suggest addressing heightened levels of anxiety as an effective way to decrease problem behaviors, it might be more effective to identify mediators of the associations between problem behaviors and self-reported state anxiety rather than those between problem behaviors and self-re- ported trait anxiety. Relatedly, targeted interventions training youths with ASD with high self-reported state anxiety to apply adaptive emotion regulation strategies (e.g., cognitive reappraisal) in stressful situations may be more effective than targeting individuals with high self-reported trait anxiety. However, as we cannot draw any temporal or causal conclusions in this study, we should not rule out the possibility that exhibiting problem behaviors put youths with ASD in situations that heighten their state anxiety. Future studies should use a controlled experimental design to investigate the causal relationships between state anxiety and different problem behaviors in individuals with ASD to inform interventions that support the cognitive-emotional and behavioral development of youths with ASD.

\section{Limitations}

There are several limitations to be considered when interpreting the findings of this study. First, the majority of participants had subclinical levels of anxiety, at least as shown in their STAI scores. Although there is no absolute cut-off score for the STAI, typically, individuals with anxiety disorder would score in the range of $47-61$ in the STAIT ${ }^{68}$ while the mean STAIT score for the participants in this study was 38.05. Second, we could not conduct analyses of the effects of gender because we had only five female participants. Finally, as a correlational study, we cannot make claims about causal relationships between anxiety and behavioral variables.

\section{Conclusion}

Self-reported state and trait anxiety and parent-reported trait anxiety were each associated with different sets of demographic and behavioral variables, while self-reported state anxiety and parent-reported trait anxiety showed relatively similar association patterns. Emphasizing that self-reported state and trait anxiety and parent-reported trait anxiety are operating under distinct underlying mechanisms, we urge future researchers who study anxiety of adolescents with ASD to be aware of the instruments' differences. The findings also highlight the need for future studies exploring precisely what types of anxiety constructs are captured by items in each instrument. Finally, more studies investigating the causal associations between state anxiety and problem behaviors and mediators or moderators of the associations are needed to inform the development of effective cognitive-emotional and behavioral interventions that teach adaptive strategies to youths with ASD.

\section{Supplementary Materials}

The online-only Data Supplement is available with this article at https://doi.org/10.30773/pi.2020.0328. 


\section{Acknowledgments}

We acknowledge that this work was supported by Seoul National University Research Grant (No. 02-2017-034), the Institute of Information \& Communications Technology Planning \& Evaluation (IITP) grant funded by the Korea government (MSIT) (2019-0-00330) and Original Technology Research Program for Brain Science of NRF funded by the Korean government (MSIT) (NRF-2017M3C7A1027467).

\section{Conflicts of Interest}

The authors have no potential conflicts of interest to disclose.

\section{Author Contributions}

Conceptualization: So Yoon Kim, Hee Jeong Yoo. Data curation: So Yoon Kim, Joo Hyun Kim, Guiyoung Bong. Formal analysis: So Yoon Kim. Funding acquisition: Hee Jeong Yoo. Investigation: So Yoon Kim. Methodology: So Yoon Kim. Project administration: Guiyoung Bong, Joo Hyun Kim, Jong-myeong Kim. Supervision: Hee Jeong Yoo. Writing_original draft: So Yoon Kim, Young Ah Kim, Da-Yea Song. Writing_review \& editing: So Yoon Kim, Young Ah Kim, Da-Yea Song, Jong-myeong Kim, Joo Hyun Kim, Hee Jeong Yoo.

\section{ORCID iDs}

$\begin{array}{ll}\text { So Yoon Kim } & \text { https://orcid.org/0000-0003-1349-8031 } \\ \text { Young Ah Kim } & \text { https://orcid.org/0000-0003-4166-4447 } \\ \text { Da-Yea Song } & \text { https://orcid.org/0000-0002-7144-4739 } \\ \text { Guiyoung Bong } & \text { https://orcid.org/0000-0001-8630-9399 } \\ \text { Jong-myeong Kim } & \text { https://orcid.org/0000-0002-6917-9358 } \\ \text { Joo Hyun Kim } & \text { https://orcid.org/0000-0002-9000-0092 } \\ \text { Hee Jeong Yoo } & \text { https://orcid.org/0000-0003-0521-2718 }\end{array}$

\section{REFERENCES}

1. Gotham K, Bishop SL, Hus V, Huerta M, Lund S, Buja A, et al. Exploring the relationship between anxiety and insistence on sameness in autism spectrum disorders. Autism Res 2013;6:33-41.

2. Howlin P, Mawhood L, Rutter M. Autism and developmental receptive language disorder--a follow-up comparison in early adult life. II: Social, behavioural, and psychiatric outcomes. J Child Psychol Psychiatry 2000;41:561-578.

3. Leyfer OT, Folstein SE, Bacalman S, Davis NO, Dinh E, Morgan J, et al. Comorbid psychiatric disorders in children with autism: interview development and rates of disorders. J Autism Dev Disord 2006;36:849-861.

4. Wood JJ, Drahota A, Sze K, Har K, Chiu A, Langer DA. Cognitive behavioral therapy for anxiety in children with autism spectrum disorders: a randomized, controlled trial. J Child Psychol Psychiatry 2009;50: 224-234.

5. van Steensel FJ, Bögels SM, Perrin S. Anxiety disorders in children and adolescents with autistic spectrum disorders: a meta-analysis. Clin Child Fam Psychol Rev 2011;14:302-317.

6. Joshi G, Petty C, Wozniak J, Henin A, Fried R, Galdo M, et al. The heavy burden of psychiatric comorbidity in youth with autism spectrum disorders: a large comparative study of a psychiatrically referred population. J Autism Dev Disord 2010;40:1361-1370.

7. Reaven J. The treatment of anxiety symptoms in youth with high-functioning autism spectrum disorders: developmental considerations for parents. Brain Res 2011;1380:255-263.

8. Kerns CM, Kendall PC, Zickgraf H, Franklin ME, Miller J, Herrington J. Not to be overshadowed or overlooked: functional impairments associated with comorbid anxiety disorders in youth with ASD. Behav Ther 2015;46:29-39.

9. Rodgers J, Riby DM, Janes E, Connolly B, McConachie H. Anxiety and repetitive behaviours in autism spectrum disorders and williams syndrome: a cross-syndrome comparison. J Autism Dev Disord 2012;42: 175-180.
10. van Steensel FJA, Heeman E. Anxiety levels in children with autism spectrum disorder: a meta-analysis. J Child Fam Stud 2017;26:17531767.

11. Vasa RA, Mazurek MO. An update on anxiety in youth with autism spectrum disorders. Curr Opin Psychiatry 2015;28:83-90.

12. Gotham K, Brunwasser SM, Lord C. Depressive and anxiety symptom trajectories from school age through young adulthood in samples with autism spectrum disorder and developmental delay. J Am Acad Child Adolesc Psychiatry 2015;54:369-376.e363.

13. Sukhodolsky DG, Scahill L, Gadow KD, Arnold LE, Aman MG, McDougle CJ, et al. Parent-rated anxiety symptoms in children with pervasive developmental disorders: frequency and association with core autism symptoms and cognitive functioning. J Abnorm Child Psychol 2008;36:117-128.

14. Vasa R, Kalb L, Mazurek M, Kanne S, Freedman B, Keefer A, et al. Agerelated differences in the prevalence and correlates of anxiety in youth with autism spectrum disorders. Res Autism Spectr Disord 2013;7:13581369.

15. Baribeau DA, Vigod S, Pullenayegum E, Kerns CM, Mirenda P, Smith IM, et al. Repetitive behavior severity as an early indicator of risk for elevated anxiety symptoms in autism spectrum disorder. J Am Acad Child Adolesc Psychiatry 2020;59:890-899.e893.

16. Tantam D. The challenge of adolescents and adults with Asperger syndrome. Child Adolesc Psychiatr Clin N Am 2003;12:143-163.

17. American Psychiatric Association. Diagnostic and Statistical Manual of Mental Disorders (5th Ed). Washington DC: American Psychiatric Association Publishing; 2013.

18. Mazurek MO, Vasa RA, Kalb LG, Kanne SM, Rosenberg D, Keefer A, et al. Anxiety, sensory over-responsivity, and gastrointestinal problems in children with autism spectrum disorders. J Abnorm Child Psychol 2013;41:165-176.

19. Wigham S, Rodgers J, South M, McConachie H, Freeston M. The interplay between sensory processing abnormalities, intolerance of uncertainty, anxiety and restricted and repetitive behaviours in autism spectrum disorder. J Autism Dev Disord 2015;45:943-952.

20. Spiker MA, Lin CE, Van Dyke M, Wood JJ. Restricted interests and anxiety in children with autism. Autism 2012;16:306-320.

21. Ooi YP, Lam C, Sung M, Tan W, Goh TJ, Fung D, et al. Effects of cognitive-behavioral therapy on anxiety for children with high-functioning autistic spectrum disorders. Singapore Med J 2008;49:215-220.

22. Ambler P, Eidels A, Gregory C. Anxiety and aggression in adolescents with autism spectrum disorders attending mainstream schools. Res Autism Spectr Disord 2015;18:97-109.

23. Kim JA, Szatmari P, Bryson SE, Streiner DL, Wilson FJ. The prevalence of anxiety and mood problems among children with autism and Asperger syndrome. Autism 2000;4:117-132.

24. Kuusikko S, Pollock-Wurman R, Jussila K, Carter AS, Mattila ML, Ebeling $\mathrm{H}$, et al. Social anxiety in high-functioning children and adolescents with Autism and Asperger syndrome. J Autism Dev Disord 2008; 38:1697-1709.

25. Niditch LA, Varela RE, Kamps JL, Hill T. Exploring the association between cognitive functioning and anxiety in children with autism spectrum disorders: the role of social understanding and aggression. J Clin Child Adolesc Psychol 2012;41:127-137.

26. Little S, Garber J. Aggression, depression, and stressful life events predicting peer rejection in children. Dev Psychopathol 1995;7:845-856.

27. Frick PJ, Lilienfeld SO, Ellis M, Loney B, Silverthorn P. The association between anxiety and psychopathy dimensions in children. J Abnorm Child Psychol 1999;27:383-392.

28. Kantor L, Endler NS, Heslegrave RJ, Kocovski NL. Validating self-report measures of state and trait anxiety against a physiological measure. Curr Psychol 2001;20:207-215.

29. Mertens J, Zane ER, Neumeyer K, Grossman RB. How anxious do you think I am? Relationship between state and trait anxiety in children with and without ASD during social tasks. J Autism Dev Disord 2017; 
47:3692-3703.

30. Yoo HJ, Bahn G, Cho IH, Kim EK, Kim JH, Min JW, et al. A randomized controlled trial of the Korean version of the PEERS $\left({ }^{\circledR}\right)$ parent-assisted social skills training program for teens with ASD. Autism Res 2014;7:145-161.

31. Lord C, Luyster R, Gotham K, Guthrie W. Autism Diagnostic Observation Schedule, Second Edition (ADOS-2) Manual. Torrance, CA: Western Psychological Services; 2012.

32. Yoo H, Bong G, Kwak Y, Lee M, Cho S, Kim B, et al. Korean Version Of Autism Diagnostic Observation Schedule-Second Edition (ADOS-2). Seoul: Inpsyt; 2017.

33. Rutter M, A. LC, Lord C. Autism Diagnostic Interview-Revised. Los Angeles, CA: Western Psychological Services; 2003.

34. Yoo H, Park K, Kwak Y, Cho S, Ban G, Kim BN. Korean Version Of Autism Diagnostic Interview-Revised (ADI- R). Seoul: Inpsyt; 2007.

35. Rutter M, Bailey A, Lord C. The Social Communication Questionnaire. Los Angeles: Western Psychological Services; 2003.

36. Constantino J, Gruber C. Social Responsiveness Scale Second Edition (SRS-2): Manual. Torrance, CA: Western Psychological Services; 2012.

37. Spielberger C. Manual for the State-Trait Anxiety Inventory (STAI). Palo Alto, CA: Counsulting Psychologists Press; 1983.

38. Achenbach T, Rescorla L. Manual for the ASEBA School-age Forms \& Profiles: An Integrated System of Multi-informant Assessment. Burlington, VT: ASEBA; 2001.

39. Cho S, Choi J. Development of the Korean form of the State-trait Anxiety Inventory for Children. Seoul J Psychiatry 1989;14:150-157.

40. Achenbach TM, Dumenci L, Rescorla LA. DSM-oriented and empirically based approaches to constructing scales from the same item pools. J Clin Child Adolesc Psychol 2003;32:328-340.

41. Kim YA, Lee J, Moon SJ, Kim Y, Oh KJ. Standardization study for the Korean version of the child behavior checklist for ages 1.5-5. Korean J Clin Psychol 2009;28:117-136.

42. Bölte S, Poustka F, Constantino JN. Assessing autistic traits: cross-cultural validation of the social responsiveness scale (SRS). Autism Res 2008;1:354-363.

43. Torres EB, Rai R, Mistry S, Gupta B. Hidden aspects of the research ADOS are bound to affect autism science. Neural Comput 2020;32:515561.

44. Park K. KEDI-WISC Manual. Seoul: Korean Educational Institute; 1991.

45. Wechsler D. Wechsler Intelligence Scale for Children-Fourth Edition (WISC-IV). San Antonio, TX: The Psychological Corporation; 2003.

46. Hwang S, Kim J, Park G, Chey J, Hong S. K-WAIS-IV Administration and Scoring Manual. Daegu: Korean Psychology; 2012.

47. Wechsler D. Wechsler Adult Intelligence Scale-Fourth Edition (WAISIV). San Antonio, TX: Pearson; 2008.

48. Kwak K, Oh S, Kim C. Korean-Wechsler Intelligence Scale for Children. 4th Ed. Seoul: Hakjisa; 2011.

49. Caci HM. "Cortesti: Stata module to test equality of two correlation coefficients." Statistical Software Components. S407302, Boston College Department of Economics, revised 07 Nov, 2000. (https://ideas.repec. org/c/boc/bocode/s407302.html)

50. Cohen J. Statistical Power Analysis for the Behavioral Sciences (2nd Ed.). Hillsdales NJ: Lawrence Erlbaum Associates; 1988.

51. Legrand LN, McGue M, Iacono WG. A twin study of state and trait anxiety in childhood and adolescence. J Child Psychol Psychiatry 1999;40: 953-958.

52. Conner CM, Maddox BB, White SW. Parents' state and trait anxiety: relationships with anxiety severity and treatment response in adolescents with autism spectrum disorders. J Autism Dev Disord 2013;43: 1811-1818.

53. Boulter C, Freeston M, South M, Rodgers J. Intolerance of uncertainty as a framework for understanding anxiety in children and adolescents with autism spectrum disorders. J Autism Dev Disord 2014;44:13911402.

54. Chamberlain PD, Rodgers J, Crowley MJ, White SE, Freeston $\mathrm{MH}$, South M. A potentiated startle study of uncertainty and contextual anxiety in adolescents diagnosed with autism spectrum disorder. Mol Autism 2013;4:31.

55. Hallett V, Lecavalier L, Sukhodolsky DG, Cipriano N, Aman MG, McCracken JT, et al. Exploring the manifestations of anxiety in children with autism spectrum disorders. J Autism Dev Disord 2013;43:23412352.

56. Gillott A, Furniss F, Walter A. Anxiety in high-functioning children with autism. Autism 2001;5:277-286.

57. Zandt F, Prior M, Kyrios M. Repetitive behaviour in children with high functioning autism and obsessive compulsive disorder. J Autism Dev Disord 2007;37:251-259.

58. Hanft B, Miller L, Lane S. Toward a consensus in terminology in sensory integration theory and practice: Part 3: Observable behaviors: sensory integration dysfunction. Sensory Integration Special Interest Section Quarterly 2000;23:1-4.

59. Top Jr DN, Luke SG, Stephenson KG, South M. Psychophysiological arousal and auditory sensitivity in a cross-clinical sample of autistic and non-autistic anxious adults. Front Psychiatry 2019;9:783.

60. Engel-Yeger B, Dunn W. The Relationship between sensory processing difficulties and anxiety level of healthy adults. Br J Occup Ther 2011;74: 210-216.

61. Hofmann SG, Bitran S. Sensory-processing sensitivity in social anxiety disorder: relationship to harm avoidance and diagnostic subtypes. J Anxiety Disord 2007;21:944-954.

62. McMahon K, Anand D, Morris-Jones M, Rosenthal MZ. A path from childhood sensory processing disorder to anxiety disorders: the mediating role of emotion dysregulation and adult sensory processing disorder symptoms. Front Integr Neurosc 2019;13:22-22.

63. Green SA, Ben-Sasson A, Soto TW, Carter AS. Anxiety and sensory overresponsivity in toddlers with autism spectrum disorders: bidirectional effects across time. J Autism Dev Disord 2012;42:1112-1119.

64. Van Hecke A, Lebow J, Bal E, Lamb D, Harden E, Kramer A, et al. Electroencephalogram and heart rate regulation to familiar and unfamiliar people in children with autism spectrum disorders. Child Dev 2009;80: 1118-1133.

65. Jain N AS, Bozdag S, Dolan BK, McVey AJ, Willar KS, Pleiss SS, et al. Have it, know it, but don't show it: Examining Physiological arousal, anxiety, and facial expressions over the course of a social skills intervention for autistic adolescents. BioRxiv; 2019.

66. Poquérusse J, Pastore L, Dellantonio S, Esposito G. Alexithymia and autism spectrum disorder: a complex relationship. Front Psychology 2018; 9:1196.

67. Hurtig T, Kuusikko S, Mattila ML, Haapsamo H, Ebeling H, Jussila K, et al. Multi-informant reports of psychiatric symptoms among highfunctioning adolescents with Asperger syndrome or autism. Autism 2009;13:583-598.

68. Antony M, Orsillo S, Roemer L. Practitioner's Guide to Empirically Based Measures of Anxiety. New York: Kluwer Academic; 2001. 
Supplementary Table 1. Zero-order correlation matrix

\begin{tabular}{lccc}
\hline \multicolumn{1}{c}{ Variables } & STAIS & STAIT & CBCL anxiety \\
\hline Withdrawn/depressed & $0.40^{\dagger}$ & $0.21^{*}$ & $0.62^{\dagger}$ \\
Somatic complaints & $0.24^{*}$ & 0.20 & $0.46^{\dagger}$ \\
Rule-breaking behavior & 0.09 & -0.02 & $0.30^{\dagger}$ \\
Aggressive behavior & $0.22^{*}$ & 0.13 & $0.48^{\dagger}$ \\
SRS-2 mannerism & $0.34^{\dagger}$ & $0.31^{\dagger}$ & $0.51^{\dagger}$ \\
RRBs total scores & 0.08 & 0.08 & 0.04 \\
from the ADI-R & & & \\
ADI-R 1 & -0.08 & -0.13 & -0.12 \\
ADI-R 2 & $0.37^{\dagger}$ & $0.25^{*}$ & 0.15 \\
ADI-R 3 & -0.03 & 0.10 & -0.07 \\
ADI-R 4 & 0.01 & -0.01 & 0.16 \\
Sensory sensitivity & $0.28^{\dagger}$ & $0.21^{*}$ & $0.38^{\dagger}$ \\
\hline
\end{tabular}

$\mathrm{N}=96$ for all correlations. ${ }^{*} \mathrm{p}<0.05,{ }^{\dagger} \mathrm{p}<0.01$. ADI-R 1: Encompassing preoccupation or circumscribed pattern of interest, ADI-R 2: Compulsive adherence to routines or rituals, ADI-R 3: Motor mannerisms, ADI-R 4: Preoccupation with parts of objects or non-functional elements of material, STAIT: State-Trait Anxiety Inventory-Trait, STAIS: State-Trait Anxiety Inventory-State, CBCL: Child Behavior Checklist 